\title{
The anorectal exam is unnecessary!
}

\author{
Ralph J. Marino $\mathbb{1}^{1}$
}

Received: 17 September 2017 / Accepted: 25 September 2017

(C) International Spinal Cord Society 2018

The anorectal examination is an unnecessary part of the International Standards for Neurological Classification of Spinal Cord Injury (ISNCSCI) in almost all cases. Its requirement in the ISNCSCI is based on limited data and a modification of the "sacral sparing" definition of SCI proposed by Waters [1]. The reliability of deep anal sensation and voluntary anal contraction (VAC) are modest, and the validity of deep anal pressure (DAP) as an indicator of somatic sensation has been questioned. Finally, the predictive validity of "sacral sparing" as defined in the ISNCSCI is limited.

The basis of the inclusion of DAP and VAC in the ISNCSCI is said to be from Waters [1], who reported that a change in classification of completeness was unidirectional using his proposed sacral sparing definition, and bidirectional using the Frankel scale definition (sparing of sensory or motor function more than three levels below the neurological level of injury, NLI). Waters [1], however, did not include DAP in his definition of sacral sparing, and VAC was not the sole criterion for motor sparing! He defined motor sparing as motor function in the VAC or the toe flexor (TF) muscles. Sensory sacral sparing was defined as "presence of sensation in the perinium at the anal mucocutaneous junction, glans penis or clitoris [1]." The article did not describe which component(s) were present in persons classified as incomplete by sacral sparing.

The value of the anorectal examination in whole or in part has been questioned. The European Multicenter Study on Human Spinal Cord Injury (EM-SCI) group looked at the ability to ambulate at 1 year based on the ISNCSCI sacral sparing definition and components of the sacral exam. They found that the combination of VAC and S4-5 light touch (LT) and pin prick (PP) scores (omitting DAP) was better at predicting ambulation by 1 year than the current

Ralph J. Marino

ralph.marino@jefferson.edu

1 Sidney Kimmel Medical College at Thomas Jefferson University, 132 South 10th Street, Suite 375 Main Building, Philadelphia, PA, USA definition of incomplete (including DAP) [2]. To be fair, however, the sacral sparing criteria was meant to identify inujries that have no sparing of sensory or motor function from those with some sparing. There was no suggestion that having any sacral sparing would be a good predictor of ambulation.

The value of the sacral sparing definition of completeness is not clear, however. There are discrepencies in the description and terminology for the anorectal exam, including whether to apply pressure against the anal sphincter, the rectal wall, or both. Stimulating the rectal wall may activate autonomic pathways and generate a response not based on somatically mediated sensation [3]. Report of a large number of subjects in the control group of a clinical trial converting from complete to incomplete based solely on anorectal sensation [4] highlights the importance of assuring that the test for sacral sensation be done consistently and reflect somatic sensory preservation.

Potential alternatives exist for the anorectal exam components, DAP, VAC, and bulbocavernosus reflex. The majority of persons classified as American Spinal Injury Association impairment scale (AIS) B have LT and/or PP preservation. Zariffa looked at patterns of sacral sparing and found that for all exams at all time points in the EMSCI database at the time $(n=3848$, NLI C4-T12), only $5 \%$ had discrepant DAP and S4-5 LT-PP results [5]. For those classified as AIS B, the rate was higher, $22 \%$, with $12.5 \%$ having just DAP and 9.5\% just S4-5 sensation. Marino [6] showed that pressure sensation at the S3 sensory point (S3P) may be a good substitute for DAP. Test-retest reliability of S3P was excellent $(\mathrm{kappa}=0.98)$ and agreement between DAP and S3P was $89 \%$, with differences split evenly. S3P was more sensitive than LT or PP at S4-5, present more often, and at a similar frequency as DAP.

The author has looked at the hip adductors and/or TFs (HA-TF) as an alternative to VAC in persons with SCI above L1. Motor function in the HA-TF and VAC had high agreement ( 46 out of 49 , kappa $=0.81$ ). In the three cases where these differed, HA-TF was present with no VAC (unpublished data). This suggests that the HA-TF, neither 
required elements of the ISNCSCI, may be more sensitive to sparing of motor function than VAC.

This leaves the bulbocavernosus (BC) reflex, an optional element, and also unnecessary. The anocutaneous (anal wink) reflex can and should be performed while checking $\mathrm{PP}$ sensation at S4-5. This is a monosynaptic reflex quite similar to the $\mathrm{BC}$ reflex. To my knowledge, there are no studies comparing the anal wink and $\mathrm{BC}$ reflex for prediction of bowel or bladder function. The presence of the anocutaneous reflex has been associated with bladder continence in children with myelomeningocele [7].

All of this, however, misses the point. The rationale for performing the anorectal exam, as described in the anorectal exam module of InSTeP, is that a positive result provides "evidence that some nerve transmission is getting past the injured part of the spinal cord [8]." The previous definition of incomplete, sparing more than three levels below the neurological level of injury, may have been too lenient. In some cases, the zone of partial preservation (ZPP) likely extended more than three levels below the NLI. Recovery of segments adjacent to the NLI without revovery below the lower edge of the ZPP resulted in persons with SCI converting to "complete" due to improvement in the NLI and resultant shrinkage of the ZPP to less than three segments. Conversely, the sacral sparing definition may be too strict. A person with a cervical SCI who can activate a lower extremity muscle has evidence of nerve transmission past the site of the injured cord irrespective of the status of DAP or VAC.

Already, the Neurological Standards Committee has moved toward targeted performance of the anorectal exam. The 2011 version of the standards states "in patients who have LT or PP sensation at S4-5, evaluation of DAP is not necessarily required as the patient already has a designation for a sensory incomplete injury [9]." It is not clear why the committee did not use the same reasoning with VAC: if a patient is sensory incomplete with motor sparing more than three levels below the motor level, then this patient already has a designation of motor incomplete, and testing VAC is unnecessary. As incomplete injuries are increasing in frequency, the majority of patients with SCI will have sensory and or motor function clearly below the level of spinal cord damage. When information about somatic sacral sparing is needed, testing of $\mathrm{S} 3$ pressure and TFs/HAs will provide similar if not more valid information than DAP or VAC testing. The exception to this would be in cases where the area of spinal cord injury and the sacral components coincide, namely conus or cauda equina injuries. In all other cases, the anorectal exam is simply not needed.

\section{Compliance with ethical standards}

Conflict of interest The author declares no competing interests.

\section{References}

1. Waters RL, Adkins RH, Yakura JS. Definition of complete spinal cord injury. Paraplegia. 1991;29:573-81.

2. Van Middendorp JJ, Hosman AJF, Pouw MH, Van De Meent H. Is determination between complete and incomplete traumatic spinal cord injury clinically relevant validation of the ASIA sacral sparing criteria in a prospective cohort of 432 patients. Spinal Cord. 2009;47:809-16

3. Wietek BM, Baron $\mathrm{CH}$, Erb M, Hinninghofen $\mathrm{H}$, Badtke A, Kaps HP, et al. Cortical processing of residual ano-rectal sensation in patients with spinal cord injury: an fMRI study. Neurogastroenterol Motil. 2008;20:488-97.

4. Lammertse DP, Jones LA, Charlifue SB, Kirshblum SC, Apple DF, Ragnarsson KT, et al. Autologous incubated macrophage therapy in acute, complete spinal cord injury: results of the phase 2 randomized controlled multicenter trial. Spinal Cord. 2012;50:661-71.

5. Zariffa J, Kramer JLK, Jones LAT, Lammertse DP, Curt A, Steeves JD. Sacral sparing in SCI: beyond the S4-S5 and anorectal examination. Spine J. 2012;12:389-400.

6. Marino RJ, Schmidt-Read M, Kirshblum SC, Dyson-Hudson TA, Tansey K, Morse LR, Graves DE. Reliability and validity of S3 pressure sensation as an alternative to deep anal pressure in neurological classification of persons with spinal cord injury. Arch Phys Med Rehabil. 2016;97:1642-6.

7. Sanders C, Driver CP, Rickwood AM. The anocutaneous reflex and urinary continence in children with myelomeningocele. BJU Int. 2002;89:720-1.

8. American Spinal Injury Association. InSTeP Module 4 - The anorectal exam. https://lms3.learnshare.com/home.aspx. Accessed 4 Sep 2017.

9. Kirshblum SC, Burns SP, Biering-Sorensen F, Donovan W, Graves DE, Jha A, et al. International standards for neurological classification of spinal cord injury (revised 2011). J Spinal Cord Med. 2011;34:535-46. 